Tecnociencia, Vol. 22, $\mathrm{N}^{\circ} 2:$ 205-225

julio-diciembre 2020

\title{
DIVERSIDAD ALPHA Y BETA DE ABEJAS EUGLOSSINI (HYMENOPTERA: APIDAE) EN EL DOSEL Y SOTOBOSQUE DEL CERRO TUREGA, PROVINCIA DE COCLÉ, PANAMÁ
}

\author{
${ }^{1}$ Karla Y. Reyes Ledezma, ${ }^{* 2}$ Alonso Santos Murgas, ${ }^{3}$ Pedro González, ${ }^{4}$ Iris \\ Y. Gómez \& Aniceto Barrios Vargas \\ 1,2,3,4,5 Universidad de Panamá, Facultad de Ciencias Naturales Exactas y Tecnología; \\ Centro Regional Universitario de Coclé, Departamento de Zoología. \\ ${ }^{2}$ Universidad de Panamá, Facultad de Ciencias Naturales Exactas y Tecnología; Centro \\ Regional Universitario de Coclé, Museo de Invertebrados G. B. Fairchild. \\ 1,2,3,4,5 Sociedad Mesoamericana Para La Biología y la Conservación-Capítulo de \\ Panamá. \\ E-mail: $\quad$ santosmurgasa@gmail.com; alonso.santos@up.ac.pa ; \\ karla_2412@hotmail.com; pedroar1400@gmail.com; iyg0123@gmail.com ; \\ abarrios39@hotmail.com \\ *Autor de Correspondencia (Dorcid.org/0000-0001-9339-486X
}

\section{RESUMEN}

Con el objetivo de determinar la diversidad de abejas Euglossini en áreas boscosa como en áreas de cultivos de la Reserva Hídrica de Cerro Turega, provincia de Coclé, Panamá. Se realizaron colectas tanto en sotobosque como en el dosel, por medio de trampas "Bottle traps", al igual que colectas por método de zigzag, donde se usaron tres esencias para atraer las abejas y ser colectadas por medio de una red entomológica; durante 6 meses, febrero a julio de 2019. Los especímenes colectados fueron identificados a especies en el MIUP de la Universidad de Panamá. Se colectaron un total de 769 especímenes de abejas de las orquídeas, clasificados en 22 especies y cuatro géneros de euglosinas para la R.H. Cerro Turega. El índice de diversidad de Shannon-Wiener para el total de la muestra fue de $\mathrm{H}^{\prime}=2.78$. El índice de Simpsom fue de $\mathrm{D}_{\mathrm{si}}=0.74$ y una dominancia de especies de $\mathrm{D}=0.26$. Se tomó en cuenta la efectividad de los métodos utilizados para este muestreo, estableciendo que no hay diferencias significativas (Mann-Whitney $\mathrm{U}=48 ; \mathrm{P}=0.1728$ ), por lo que ambos métodos son eficientes, para este tipo de estudios. Con el método de trampas manuales se obtiene 
que el $75.23 \%$ pertenece al área abierta (cultivo), mientras que el $24.78 \%$ corresponde al área cerrada (boscosa). En cuanto a la altura de las trampas el $75.92 \%$ se colectaron en sotobosque y $24.08 \%$ en el dosel. Mientras que con el método de Zigzag, $59.9 \%$ de la muestra se colecto en área abierta (cultivos), y un $17.7 \%$ en área cerrada (boscosa). Para la R. H. Cerro Turega, la diversidad de abejas tiende a ser alta, por lo que es necesario mantener la conservación tanto de la flora y fauna en estas zonas.

\title{
PALABRAS CLAVES
}

Euglossa, Exaerete, Eulaema, Eufriesea, aceite de eucalipto, aceite de clavo, vainilla.

\section{ALPHA AND BETA DIVERSITY OF EUGLOSSINI BEES (HYMENOPTERA: APIDAE) IN THE CANOPY AND UNDERWOOD OF CERRO TUREGA, COCLE PROVINCE, PANAMA}

\begin{abstract}
In order to determine the diversity of Euglossini bees in both forest and crop areas of Cerro Turega's Water Reserve, Cocle province, Panama. We collected bees both in the underwood and in the canopy using manual traps (Bottle traps) and the zigzag method. For 6 months (february to july of 2019), three essences were used in an entomological net to attract the bees to be collected. The collected specimens were identified to species in the MIUP of the University of Panama. A total of 769 bee specimens were collected from the orchids, classified into 22 species and four genera of euglosines for Cerro Turega's Water Reserve. The Shannon-Wiener diversity index for the total sample was $\mathrm{H}^{\prime}=2.78$. The Simpsom index was $\mathrm{Dsi}=0.74$ and a species dominance was $\mathrm{D}=0.26$. We attempted to measure the effectiveness of the methods used for this sampling, therefore; results showed that there are no significant differences (MannWhitney $\mathrm{U}=48 ; \mathrm{P}=0.1728$ ), suggesting that both methods are efficient for this type of studies. With the manual traps method, we obtain that $75.23 \%$ belongs to the open area (crops), while $24.78 \%$ corresponds to the closed area (forest). As for the height of the traps, $75.92 \%$ were collected in the underwood and $24.08 \%$ in the canopy. While with the Zigzag method, $59.9 \%$ of the sample was collected in open area (crops), and $17.7 \%$ in closed area (forest). For Cerro Turega's Water Reserve, bee diversity tends to be high, so it is necessary to maintain the conservation of both flora and fauna in these areas.
\end{abstract}




\section{KEY WORDS}

Euglossa, Exaerete, Eulaema, Eufriesea, eucalyptus oil, clove oil, vanilla.

\section{INTRODUCCIÓN}

Las abejas euglosinas (Apidae: Euglossini), son abejas de colores metálicos de tamaño mediano a grande y regularmente tienen la glossa "lengua" muy larga, de allí su nombre de euglosinas (Powell y Powell 1987). Estas abejas polinizan más de 50 familias de plantas tropicales siendo muy importantes en los bosques y ecosistemas (Powell y Powell, 1987; Ramírez et al., 2002). A este grupo de abejas también se les llama abejas de las orquídeas debido a que los machos las visitan para colectar las fragancias que éstas producen, por lo que desarrollan estructuras especializadas para facilitar la polinización (Ramírez et al., 2002). También se les ha considerados como indicadoras de estado de conservación, debido a su respuesta ante los cambios en su entorno (Tonhasca et al., 2000; Brito y Rêgo, 2001; Silva y Rebêlo, 2002).

Dentro del orden Hymenoptera, la familia Apidae es una de las más numerosa y estudiada, ya que abarca a todas las abejas de comportamiento social, y otras no sociales (Roubik, 1992). La importancia de las abejas silvestres como polinizadores en los ecosistemas es muy amplia siendo ellas responsables del $80 \%$ de la polinización (Powell y Powell, 1987). Los polinizadores proveen de un servicio esencial a los ecosistemas que da como resultado la reproducción sexual de muchas plantas. Además, benefician indirectamente a la sociedad incrementando la seguridad alimentaria por medio del papel que juegan en la conservación de la diversidad biológica en ecosistemas naturales y agrícolas; las señales de la deficiencia de polinizadores son más sutiles que en la agricultura, pero en consecuencia pueden causar la extinción local de especies vegetales, escases de frutos y animales frugívoros, la pérdida de la cobertura boscosa y la degradación y muerte de ecosistemas saludables (Eardley et al., 2006). Ducke (1902) y Dressler (1982), destacaron que las abejas de las orquídeas prefieren los bosques húmedos, pero también destacaron que algunas especies habitan en sabana y bosque de galería. 
En los últimos años se ha incrementado la preocupación social sobre la calidad del ambiente, la degradación de los ecosistemas, el cambio climático y la pérdida de la biodiversidad, tanto a nivel local como regional y mundial. Desde hace varias décadas, se considera la deforestación la fragmentación y pérdida del hábitat, las principales amenazas para la biodiversidad terrestre.

De acuerdo con Samways, M. et al., (2010) las abejas han sido usadas como bioindicadores principalmente ecológicos y ambientales, ya que muestran los efectos de los cambios ambientales como la alteración del hábitat, la fragmentación o el cambio climático, respondiendo predictivamente de manera observable y cuantificable a perturbaciones o a cambios en el estado del ambiente.

Existen más de 200 especies de Euglossini descritas, 76 especies se encuentran en América Central y el Sur de México y 70 en Panamá y Costa Rica; estos dos países albergan el 38\% de todas las especies descritas de Euglossini (Roubik y Hanson, 2004). En Panamá actualmente se están realizando esfuerzos de colecta para determinar la diversidad de abejas Euglossini (Santos M., 2009; Santos M. et al., 2011; Santos M. et al., 2012; Santos M., 2014; Koo Ch. Y Santos M., 2015; Santos M. et al., 2016; Santos M. et al., 2018; Osorio A. y Santos M., 2017). Los modelos de distribución potencial de euglosinas ayudarán a establecer sitios de alta diversidad de euglosinas que aún no están bien explorados y para enfocar los esfuerzos de colecta en esas áreas.

Por ello este trabajo contribuirá a tener una aproximación de la diversidad alpha y beta de abejas de las orquídeas en el dosel y sotobosque del Cerro Turega, provincia de Coclé, Panamá; y al mismo tiempo incentivar la conservación en las áreas boscosas, ya que estas abejas son consideradas indicadoras de estado de conservación, proporcionando así un parámetro de la diversidad y riqueza presente en la provincia de Coclé. 


\section{METODOS}

Este estudio se llevó a cabo en la Reserva Hídrica de Cerro Turega, escogimos dos áreas de estudio; uno en área abierta intervenido por el ser humano y otro en área cerrada de ámbito boscoso, localizado en la Provincia de Coclé, República de Panamá.

El área abierta, dos $\mathrm{Km}^{2}$ (cultivo agrícola): comprende en su mayoría cultivos de café, plátanos, naranjos, yuca, maíz y otros cultivos artesanales que las personas del área tienen para consumo propio. Ubicado (N 08³7'09.3" y W 80¹1'27.1') a una altura de $421 \mathrm{msnm}$.

El área cerrada (bosque) y un área aproximada de cuatro $\mathrm{Km}^{2}$ : comprende vegetación de galería, vegetación en regeneración, parches y vegetación primaria. Ubicado ( $\mathrm{N} \mathrm{08} 37^{\circ} 21.7^{\prime \prime}$ y W 80¹'03.5”) con una altitud de 445 msnm (Fig. 1).

Se escogieron dos sitios uno con intervención antrópica y otro con bosque conservado; en el cual se colocaron 24 trampas manuales con botellas de plástico $(2.25 \mathrm{~L})$, se adopta una estrategia sistemática para cuantificar relaciones entre variables Walker (2005), para así determinar la abundancia y diversidad de las especies de abejas Euglossini por medio de índices estadísticos como Shannon y Simpson.

En este estudio como técnica de colecta se utilizaron trampas manuales hechas con botellas de plásticos $(2.25 \mathrm{~L})$ y $(600 \mathrm{ml})$ con cebos para abejas de las orquídeas, que consisten en esencias aromáticas como el eucalipto que embeben el algodón guindado desde las trampas al momento de colocarlas en el campo (Nemesio y Silveira, 2006).

Se utilizaron en total 18 árboles, 9 árboles en la parcela con intervención antrópica y 9 árboles en la parcela con bosque conservado; siendo un total de 24 trampas manuales con botellas, en el cual 12 trampas estuvieron fijas en cada parcela durante 15 días, 6 en el dosel y 6 en el sotobosque, mientras que las otras 12 fueron trampas con polea colocadas aleatoriamente entre el dosel y el sotobosque cada 15 días. 


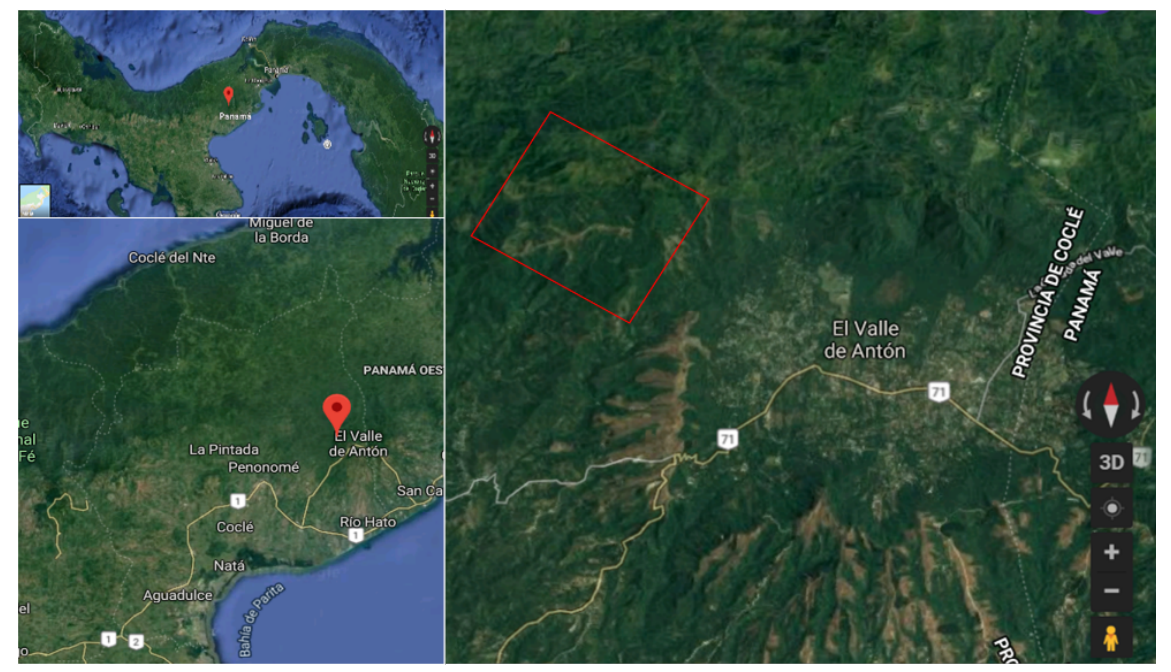

Fig. 1 Fragmento de Bosque, Cerro Turega, Penonomé. Fuente: Googlemap.com, 2019

La distancia que separó las trampas fijas fue de aproximadamente 40 metros en cada árbol fijo y las aleatorias fueron de mínimo 25 metros. Las trampas manuales aleatorias se revisaron 2 horas en la mañana (8:00 am - 10:00 am) y 2 horas en la tarde (2:00 pm - 4:00 pm) en cada zona de muestreo.

También se realizaron colectas manuales utilizando la técnica de "zigzag", la cual se estableció un transepto de tres diagonales en las dos áreas de estudios con hilo pabilo, amarrado de extremo a extremo de cada árbol, a una altura de 1.60 a dos metros del suelo, en donde se colocaron los tres atrayentes aromáticos como aceite de clavo, vainilla y eucaliptos impregnados en algodón para atraer a los machos de euglosinas y con ayuda de una red entomológica se realizaron las capturas en el tiempo intermedio de los muestreos mencionados (Nemésio y Morato, 2006).

La muestra obtenidas por esta eficaz técnica fueron colectada dos veces al mes, durante 6 meses, los individuos colectados se preservaron en recipientes con alcohol al 70\% y rotulados; las muestras fueron llevada al Museo de Invertebrados G. B. Fairchild (MIUP), de la Universidad 
de Panamá, en el cual se realizó la identificación de los especímenes utilizando la clave de Roubik y Hanson (2004), y así los especímenes separados, fueron montados con alfileres $\mathrm{N}^{\circ} .1$ y rotulados para posteriormente ser colocados en cajas entomológica.

Los datos recolectados en cada gira de muestreo fueron colocados en una base de datos de Microsoft Excel 2016. Posteriormente fueron analizados en porcentajes por número de colectas de métodos usados y géneros identificados. Los datos obtenidos en los 6 meses de muestreo fueron analizados en el programa GraphPad Prisma 7.0, con pruebas no paramétricas de Mann Whitney test, para comparar las áreas de estudios y la efectividad de los métodos utilizados.

\section{RESULTADOS}

Durante las 12 giras de campo realizadas a la Reserva Hídrica de Cerro Turega se colectaron 769 individuos de abejas de las orquídeas, que posteriormente fueron identificadas en el laboratorio. Los cuatro géneros encontrados para Reserva Hídriga de Cerro Turega son: Euglossa, Exaeretes, Eulaema y Eufriesia.

La muestra total identificada durante los seis meses de muestreo el 91.8\% ( $\mathrm{n}=706)$ del total corresponde al género Euglossa, siendo el género más abundante, el 5.5\% $(\mathrm{n}=42)$ al género Eulaema, $1.8 \%(\mathrm{n}=14)$ al género Exaerete y el $0.9 \%(\mathrm{n}=7)$ correspondientes al género Eufriesia (Fig. 2). 


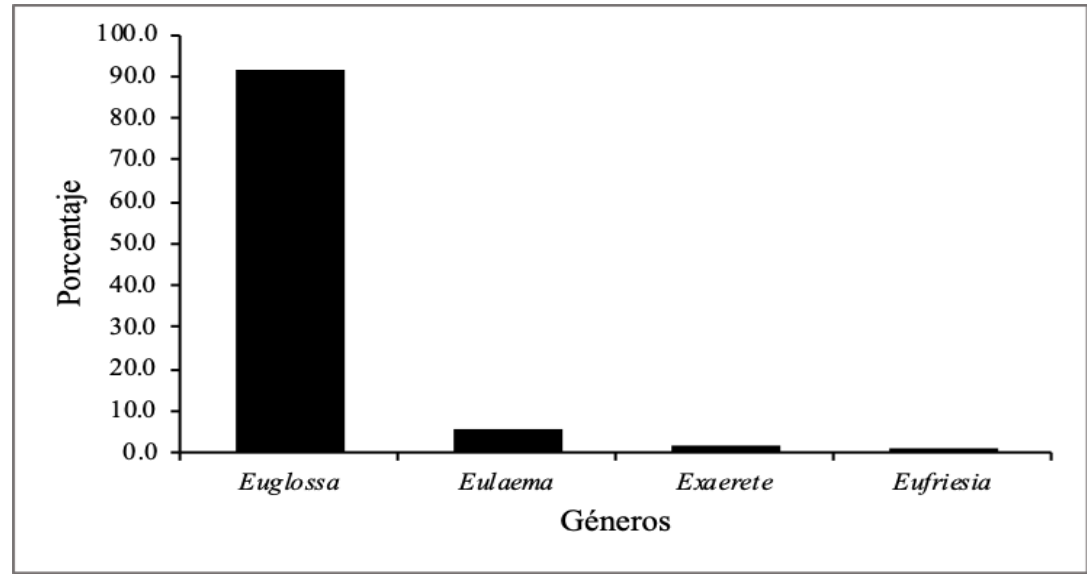

Fig. 2 Porcentaje de abundancia de géneros de la tribu Euglossini, colectados en el periodo de muestreo en la RHCT

Del género Euglossa se identificaron 14 especies, en cuanto al género Eulaema, se registraron cuatro especies y del género Exaerete y Eufriesia se identificaron dos especies para cada uno de estos (Fig. 3).

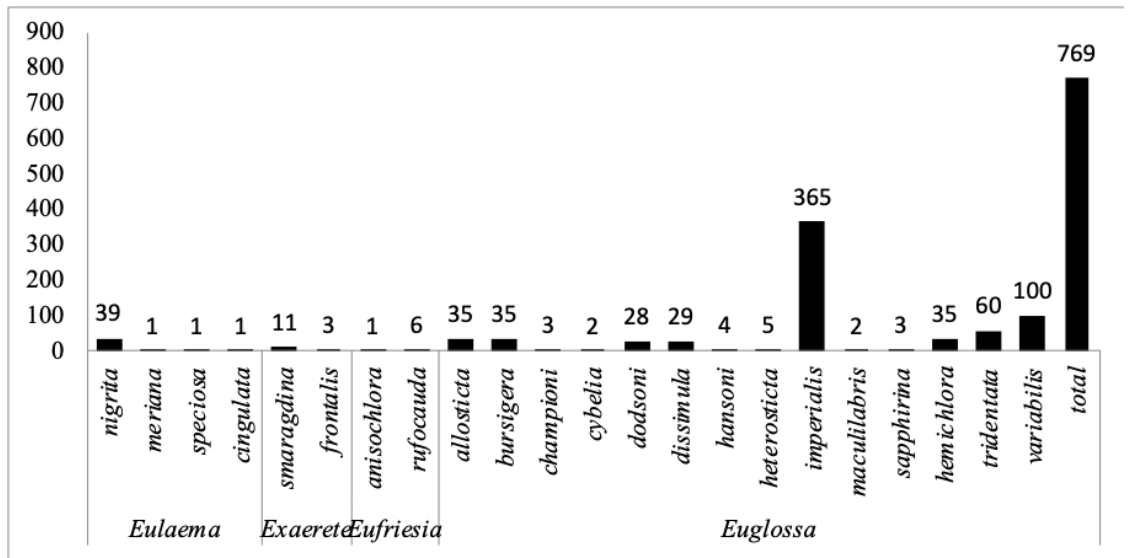

Fig. 3 Cantidad total de especies registradas por género de la Tribu Euglossini en la RHCT 
El índice de diversidad de Shannon-Wiener arrojó un resultado de $\mathrm{H}^{\prime}=2.78$ dando una alta diversidad de especies en la Reserva Hídrica de Cerro Turega. De igual forma se utilizó el índice de Simpsom resultando un índice de $\mathrm{D}_{\mathrm{si}}=0.74$ (Cuadro 1).

Cuadro 1. Índices de diversidad de abejas de las Orquídeas presentes en La RHT

\begin{tabular}{llllllll}
\hline Meses & Febrero & Marzo & Abril & Mayo & Junio & Julio & Total \\
\hline H $^{\prime}$ & 2.75 & 2.43 & 2.19 & 2.1 & 2.09 & 1.74 & 2.78 \\
D & 0.73 & 0.86 & 0.85 & 0.88 & 0.89 & 0.89 & 0.74 \\
S & 175 & 134 & 127 & 112 & 105 & 97 & 769 \\
\% & 22.76 & 17.43 & 16.51 & 14.56 & 13.65 & 12.61 & 100 \\
\hline H'=Índice de Shannon-Wiener; D=Índice de Simpsom; S=Total de \\
especies; \%=Porcentaje
\end{tabular}

En cuanto a la diversidad de géneros y especies de acuerdo al método de muestreo, se usaron dos métodos, los cuales logramos medir la efectividad de cada uno durante el tiempo de Estudio (Cuadro 2). Los datos recolectados en ambos métodos de muestreo fueron analizados con la prueba de Mann Whitney test, en el programa Grapad Prisma 7.0, arrojando que no hay diferencias significativas entre los datos recolectados con los dos métodos (Mann-Whitney $\mathrm{U}=48 ; \mathrm{P}=0.1728$ ) (Fig. 4).

Con relación a la metodología de trampas manuales se colectaron un total de $\mathrm{N}=436$ especímenes de la tribu Euglossini. Tomando en cuenta las dos áreas muestreadas (área cerrada y área abierta), se obtiene que el $75.23 \%(\mathrm{n}=328)$ de la muestra recolectada pertenece al área cerrada, mientras que el 24.78\% $(\mathrm{n}=108)$ corresponde al área abierta. La prueba de Mann Whitney test arrojó que hay diferencia significativa en el número de muestras colectadas en ambas áreas (Mann-Whitney $\mathrm{U}=0$; $\mathrm{P}<0.001$ ), (Fig. 5). En el área abierta el género con más colectas fue Euglossa con el $88.7 \%$, seguido de Eulaema con 7.0\%, Exaeretes con $2.7 \%$ y Eufriesia con el 1.5\%. Mientras que, para el área cerrada, Euglossa obtuvo el 93.5\%, Eulaema 3.7\%, Exaeretes 1.8\% y Eufriesia 
con el $0.9 \%$.

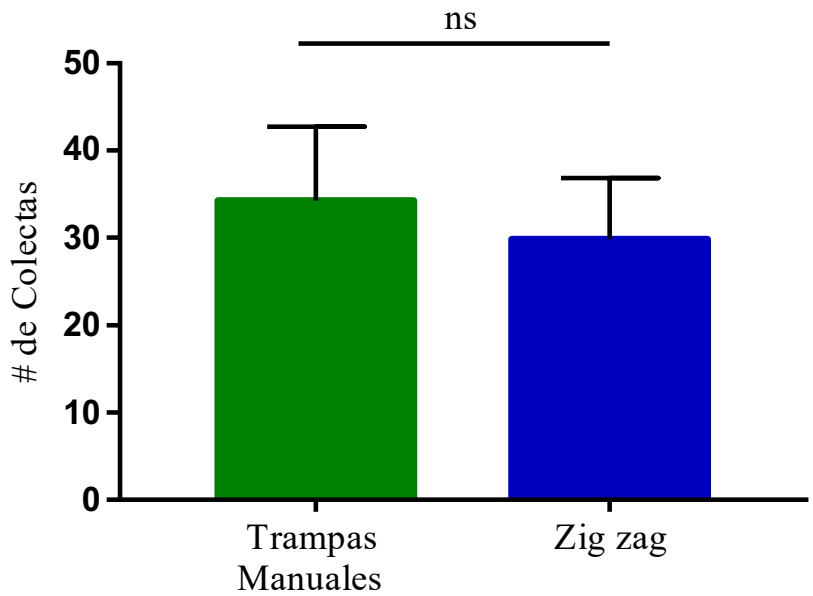

Fig. 4 Media de colectas por métodos de muestreo utilizados para la captura durante el estudio. ${ }^{\mathrm{ns}} \mathrm{p}>0.05$

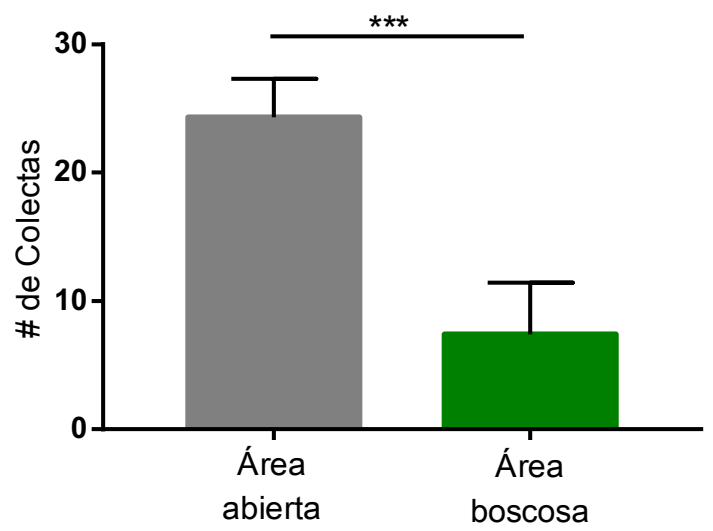

Fig. 5 Promedio de colectas comparando la cantidad de individuos en las dos áreas de estudio (abierta/boscosa). ${ }^{* * *} \mathrm{p} \leq 0.001$

Por otro lado, comparando la cantidad de individuo entre sotobosque y dosel, se obtiene que para el sotobosque se colectó un total de 75.92\% 
$(\mathrm{n}=331)$ y para el dosel fue de $24.08 \%(\mathrm{n}=105)$. Según la prueba de Mann Whitney test existe diferencia significativa en si comparamos ambos estratos (Mann-Whitney $\mathrm{U}=0 ; \quad \mathrm{P}=0.002$ ), (Fig. 6).

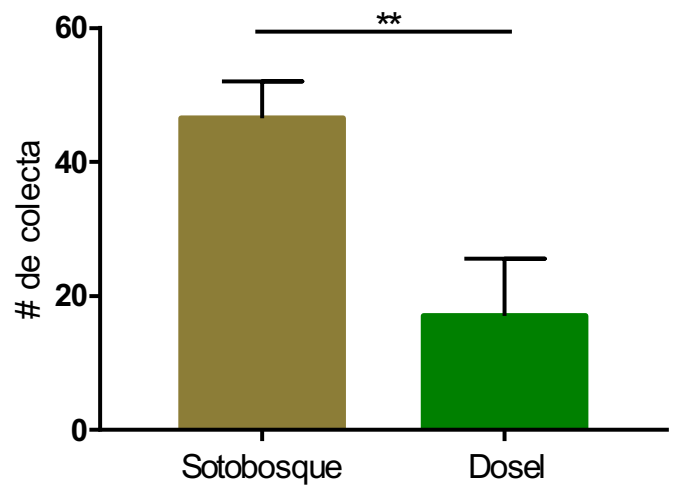

Fig. 6 Promedio de colectas comparando la cantidad de muestra por cada estrato estudiado. ${ }^{* *} \mathrm{p}=0.002$

En el sotobosque el género más colectado fue Euglossa con el 93.3\% de la muestra, Eulaema con 5.7\% y Exaeretes con $0.9 \%$. Mientras que para el dosel el género Euglossa obtuvo el 88.8\%, Eulaema 6.4\%, Exaeretes $3.0 \%$ y Eufriesia con el 1.8\%.

Con el método de Zigzag se colectó un total de 333 individuos de abejas de las orquídeas. En la cual para el área abierta de cultivos se obtuvo un porcentaje de 59.9\% de la muestra colectada, sin embargo, para el área boscosa se obtuvo el $17.7 \%$ de la muestra. También existe diferencias significativas en la cantidad de colecta en ambas áreas usando este método (Mann-Whitney $\quad \mathrm{U}=0 ; \quad \mathrm{P}=0.002$ ), (Fig. 7).

Por otro lado, tomando en cuenta los géneros colectados, el género Euglossa obtiene el 94.22\%, el género Eulaema con 4.50\%, Exaeretes con $0.90 \%$ y Eufriesia con el $0.30 \%$.

Se utilizó tres tipos de esencias como atrayentes (eucalipto, vainilla, aceite de clavo). De la cual la esencia de eucalipto fue la más efectiva 
para la colecta de estos especímenes, con el $77.18 \%$ del total de las muestras, Mientras que el $12.0 \%$ de vainilla y el $10.8 \%$ aceite de Clavo, (Fig. 8).

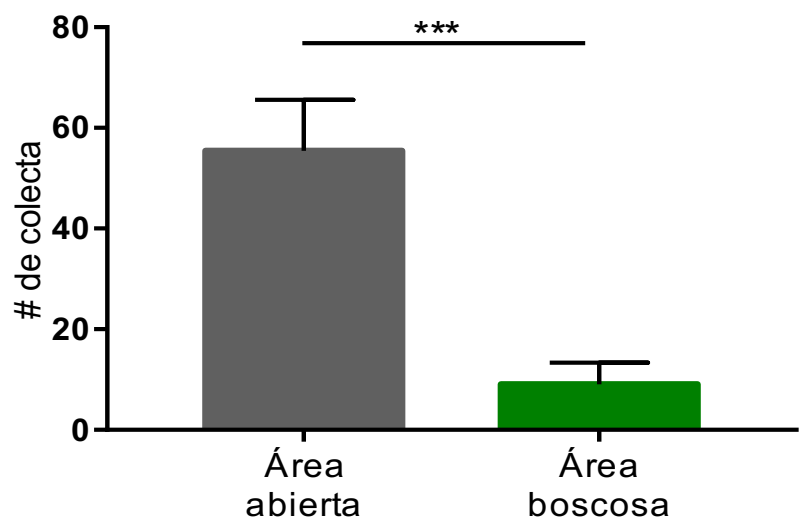

Fig. 7 Promedio de colectas comparando la cantidad de individuos, mediante el método de zigzag, en las dos áreas de estudio (abierta/boscosa). ${ }^{* *} \mathrm{p}=0.002$

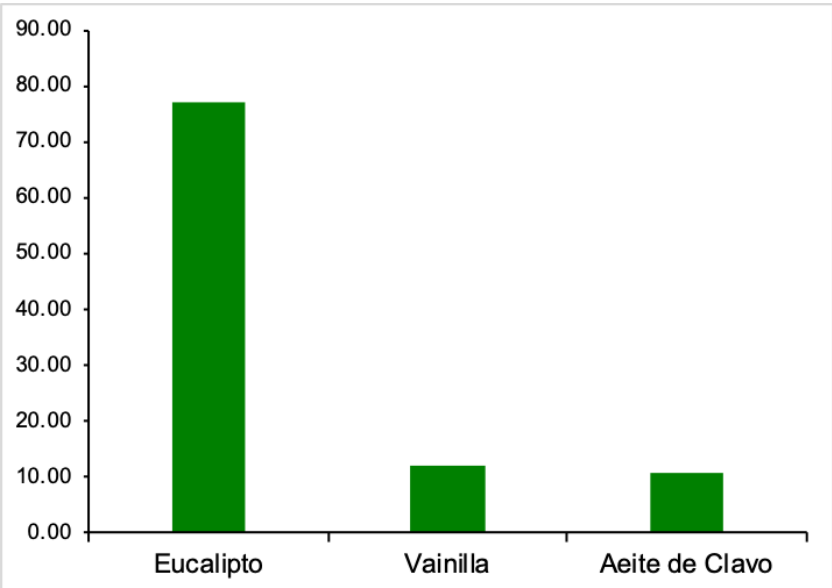

Fig. 8 Porcentaje de colecta comparando las tres esencias utilizadas para el muestreo 
Cuadro 2. Diversidad de género y especies de la Tribu Euglossini y métodos de colectas durante el periodo de muestreo.

\begin{tabular}{|c|c|c|c|c|c|}
\hline \multirow[b]{2}{*}{ Género } & \multirow[b]{2}{*}{ Especie } & \multicolumn{4}{|c|}{ Método de Colecta } \\
\hline & & TMD & TMS & MZ & Total \\
\hline \multirow[t]{4}{*}{ Eulaema } & Eulaema meriana Fig. 9 & 6 & 19 & 14 & 39 \\
\hline & Eulaema nigrita Fig. 10 & 0 & 0 & 1 & 1 \\
\hline & Eulaema speciosa & 0 & 1 & 0 & 1 \\
\hline & Eulaema cingulata & 0 & 1 & 0 & 1 \\
\hline \multirow[t]{2}{*}{ Exaerete } & Exaerete smaragdina & 1 & 7 & 3 & 11 \\
\hline & Exaerete frontalis Fig. 14 & 0 & 3 & 0 & 3 \\
\hline \multirow[t]{2}{*}{ Eufriesia } & Eufriesea anisochlora Fig. 12 & 0 & 1 & 0 & 1 \\
\hline & Eufriesea rufocauda Fig. 11 & 0 & 5 & 1 & 6 \\
\hline Euglossa & Euglossa allosticta & 5 & 18 & 12 & 35 \\
\hline \multirow[t]{13}{*}{ Fig. 13-A-B-C } & Euglossa bursigera & 7 & 22 & 6 & 35 \\
\hline & Euglossa championi & 0 & 1 & 2 & 3 \\
\hline & Euglossa cybelia & 0 & 1 & 1 & 2 \\
\hline & Euglossa dodsoni & 4 & 14 & 10 & 28 \\
\hline & Euglossa dissimula & 3 & 14 & 12 & 29 \\
\hline & Euglossa hansoni & 1 & 1 & 2 & 4 \\
\hline & Euglossa heterosticta & 2 & 2 & 1 & 5 \\
\hline & Euglossa imperialis & 43 & 131 & 191 & 365 \\
\hline & Euglossa maculilabris & 0 & 1 & 1 & 2 \\
\hline & Euglossa sapphirina & 0 & 1 & 2 & 3 \\
\hline & Euglossa hemichlora & 5 & 18 & 12 & 35 \\
\hline & Euglossa tridentata & 9 & 28 & 23 & 60 \\
\hline & Euglossa variabilis & 19 & 42 & 39 & 100 \\
\hline Total & & 105 & 331 & 333 & 769 \\
\hline
\end{tabular}

TMD: Trampas manuales de dosel; TMS: Trampa manuales de sotobosque; MZ; Método

\section{DISCUSIÓN}

Durante el periodo de muestreo entre los meses de febrero a julio de 2019, se colectaron un total de 769 especímenes de abejas Euglossini que se distribuyen en los cuatro géneros presentes en el Neotrópico (Roubik y Hanson, 2004; Koo-Chong y Santos, 2014). En este estudio el género más abundante fue Euglossa con $91.8 \%$ de la muestra total y 14 especies identificadas, esto coincide con estudios realizado en el Parque Nacional Omar Torrijos Herrera donde se colectaron 21 especies 
de dicho género representando el 68\% de la muestra (Guardia y Santos, 2014), otros estudios realizados en la Península de Azuero (Santos y Añino, 2016), al igual que en el Parque Nacional Chagres (Koo-Ch. y Santos, M. 2015).

Según los datos recopilados para las dos áreas de estudio se reportan 22 especies de euglosinas; según Roubik y Hanson (2004), existe un total de 65 especies de euglosinas en Panamá que representan el 35\% de un total de 183 especies reportadas para el Neotrópico. Lo que nos indica que se encontró, aproximadamente el $12 \%$ de las especies reportadas en Panamá.

El género que presentó mayor diversidad de especies y abundancia de especímenes, en ambos sitios, fue Euglossa. Para los dos sitios, las especies más abundantes fueron: Euglossa imperialis, Euglossa variabilis y Euglossa tridentata. Estos resultados tambien coincide con los obtenidos en el estudio de abejas de las orquídeas en la Comarca Guna de Madungandí en la provincia de Panamá (Santos, M. et al., 2012), los cuales estas especies también fueron abundantes.

El género Euglossa según autores pueden considerase sus especies como exclusivas de ambientes en aceptable o buen estado de conservación ya que se encuentran en lugares donde la diversidad en composición de especies, tamaños y formas biológicas en general fue alta y no se presentan en lugares donde el deterioro del medio es evidente (Parra, A. et al., 2016).

Euglossa imperialis (365 individuos) fue una de las especies más capturadas, esta misma especie fue la más abundante en estudios realizados en La Reserva Forestal La Tronosa, Provincia de los Santos, República de Panamá (Santos, 2009) y otros estudios realizados en un bosque tropical húmedo, en Monte Fresco, Cerro Azul, en la Provincia de Panamá (Santos, M. et al., 2011). Esto se explica porque es una especie de amplia distribución que va desde México hasta Brasil, encontrándose desde tierras bajas hasta casi los $1800 \mathrm{msnm}$, y por ser una especie generalista que busca alimento en una gran variedad de especies de orquídeas y otras plantas (Roubik \& Hanson, 2004). 
El índice de diversidad de Shannon Wiener determinó que para la R.H. Cerro Turega la diversidad es alta en los meses de verano, mientras que tiende a bajar en los meses de transición a la temporada lluviosa, por otro lado, se presentó una dominancia de las especies en particular, con el índice de Simpsom. Sin embargo, se sugiere que se hagan estudios completos y específicos en cuanto a la variación de diversidad en ambas temporadas, puesto que estos resultados dependerán de los compuestos utilizados, las condiciones de humedad y temperatura del hábitat muestreado por lo cual no es una referencia confiable en cuanto a la abundancia de ciertas especies, como sugiere Dressler (1969).

El comportamiento en cuanto a la abundancia, si mostró diferencias significativas en cuanto al número de individuos por especies, ya que fue mucho mayor en el sotobosque que en el dosel, al igual que estudios realizados en el Parque Nacional Darién, República de Panamá (Santos, 2014).

La mayoría de las especies colectadas se encontraron en el estrato del sotobosque, este comportamiento también fue reportado por otros autores donde el número de individuos por especies tiende a ser más abundante en el sotobosque (Santos et al., 2012). Lasso, E. et al., (1997), manifiestan que las abejas de las orquídeas, para evitar sobrecalentarse, se valen de diferentes técnicas de forrajeo, ya sea forrajeando a determinadas horas del día, seleccionando las áreas de forrajeo en la sombra (sotobosque), cambiando la velocidad de vuelo y la frecuencia de descansos. Es posible que las aparentes diferencias en captura por estrato puedan deberse simplemente a diferencias en estrategias de forrajeo, mencionados anteriormente.

En cuanto a la metodología de trampas de botella, es un método efectivo, es práctica y puede alcanzar diferentes estratos del bosque o para colectar durante mucho tiempo y en más sitios particularmente. Sin embargo, Veiga y Barbosa (2015), señalan que los métodos pasivos de trampas de botella son menos representativos de la diversidad de machos euglosinos presente que los muestreos activos con redes entomológicas, pero que ofrecen una alternativa eficiente para diseños 
de muestreos en varios sitios simultáneamente.

Para los dos sitios en conjunto, el atrayente más efectivo fue el aceite de eucalipto, representando más de la mitad de los individuos colectados, mientras que el atrayente menos efectivo fue el aceite de clavo, esto concuerda con los estudios de Koo-Chong y Santos (2014). Según, Armbruster y McCormick (1990) las moléculas de los aromas se dispersan mejor en un clima cálido, posiblemente la esencia de eucalipto se dispersa más rápido en ambientes tropicales como lo es el área de estudio por lo que tiene mayor nivel de atracción para los machos de abejas.

Las euglosinas como la mayoría de los insectos diurnos necesitan de la radiación solar para entrar en actividad, pero la misma en gran intensidad puede afectarles, por lo que la actividad disminuye conforme se acerca el mediodía (Barth, 1991). Por ello son más atractivas entre las 10 y 11 de la mañana (Armbruster y McCormick, 1990). Según los resultados obtenidos sugiero que las abejas no se presentaron mucho en el área cerrada debido a la sombra de la cobertura boscosa, sin embargo, para el área de cultivos o área abierta, era más efectivo utilizar los métodos ya que había mayor intensidad de la luz solar. Es importante destacar de igual forma la temporada, debido a que las abejas suelen ser activos durante todo el año, pero suelen ser más frecuentes en la temporada de lluvias, teniendo más abundancia en el mes de marzo (Orleans, 2009).

\section{AGRADECIMIENTOS}

Al Ministerio de Ambiente por ceder el permiso de colecta. A la señora Marta y su familia por abrirme las puertas de su hogar, para brindar hospedaje durante los meses de colectas en la Reserva Hídrica Cerro Turega.

\section{REFERENCIAS}

Armbruster, y McCormick, 1990. Diel foraging patterns of male euglossine bees: ecological causes and evolutionary response by plants. 
Biotropica, 25: 122-128.

Barth, F. 1991. Insects and Flowers, the Biology of a Partnership. Princenton University Press, Princenton.

Brito, C. M., y Rêgo, M. (2001). Community of male euglossini bees (hymenoptera: apidae) in a secondary forest, alcântara, ma, Brazil. Brasil. Braz. J. Biol., 61(4): 631-638.

Dressler, R. L. 1969. Species diversity of Euglossa in Panama. Ecology, 50:713- 716.

Dressler, R. L. 1982. Biology of the orchid bees (Euglossini). Annual review of ecology and systematics, 13:373-394.

Ducke, A. 1902. As Especies Paraenses do género Euglossa latr. Bol. Mus goeldi, 3:561-575.

Eardley, C., Roth, D., Clarke, J., Buchmann, S., y Gemmil, B. 2006. Pollinators and pollination: a resource book for policy and practice. African pollinator initiative. Pretonia, south africa. 92p.

Guardia, R. E., y Santos, M. A. 2014. Diversidad y estratificación vertical de abejas de las orquídeas (Hymenoptera: Euglossinae) del Parque Nacional Omar Torrijos Herrera, Coclé, Panamá. Centros 3(2): $1-16$.

Koo S., y Santos M. A. 2015. Diversidad y abundancia de las abejas de las orquídeas en los bosques nubosos del parque nacional Chagre, Panamá, República de Panamá. Centros 4: 168-187.

Lasso, E., Ortiz, P., y Villalobos, F. 1997. Influencia de la temperatura y humedad en la distribución vertical de abejas de la subfamilia Euglossini. Course Book. 71 - 73pp. 
Nemésio, A., y. Morato, E. F. 2006. The orchid-bee fauna (Hymenoptera: Apidae) of Acre state (northwestern Brazil) and a reevaluation of euglossine bait-trapping. Lundiana, 7, 59-64.

Nemesio, A.; Silveira, F. A. 2006. Edge effects on the orchidbee fauna (Hymenoptera: Apidae) at a large remnant of Atlantic rain forest in southeastern Brazil. Neotropical Entomology 35 (3): 313-323.

Orleans, 2009. Abelhas Euglossina (Hymelnoptera: Apidae) em área de restinga do nordeste do maranhão. Neotropical Entomology 38(2):186196.

Osorio Arenas M. A. y Santos Murgas A. Estudio sobre el conocimiento de la diversidad de abejas de la orquídea (Apidae: Euglossini) en la comunidad de Ustupu, Comarca Kuna Yala, Panamá. Scientia (Panamá), 2017, Vol. 28, $\mathrm{N}^{\circ}$ 1, 61-71

Parra, A. Tupac, J. Otero, P. Sandino, J. C. Ospina, R. 2016. Abejas de las orquídeas (hymenoptera: apidae: euglossini) y su importancia como polinizadoras de amplio rango en ecosistemas naturales. In book: G. Nates. (Ed.), Iniciativa Colombiana de Polinizadores, (pp.141-155). Universidad Nacional de Colombia (Sede Bogotá).

Powell, A. H., y Powell G. V. 1987. Population dynamics of male Euglossine bees in Amazonian forest fragments. Biotropica, 19:176 179.

Ramírez, S., Dressler, R. L., y Ospina, M. 2002. Abejas euglosinas (Hymenoptera: Apidae) de la Región Neotrop ical: lista do de especies con notas sobre su biología. Biota Colombiana. 3(1):7-118.

Roubik, W. D. 1992. Ecology and natural history of tropical bees. Cambridge tropical biology series. Unites States Cambridge. University press. $514 \mathrm{p}$.

Roubik, W. D., y Hanson, P. E. 2004. Abejas de orquídeas de la américa tropical: biología y guía de campo. Facio t, trad. Santo domingo de 
Heredia, Costa Rica. Instituto Nacional de Biodiversidad, Inbio, 370p.

Samways, M. J., McGeoch M. A., y Nuevo, T. R. 2010. Conservación de insectos: un manual de enfoques y métodos. Oxford University Press,

Santos, M. A. 2009. Contribución al conocimiento de las abejas Euglossini (Hymenoptera: Apidae) y la utilización de dos atrayentes químicos, en la Reserva Forestal La Tronosa, Provincia de Los Santos, Panamá. Proyecto PROBIO- Universidad de Panamá, Agencia de Cooperación Internacional del Japón. 77- 81pp.

Santos, M. A. 2014. Monitoreo de abejas de las orquídeas (Hymenoptera: Apidae) en el parque Nacional Darién, República de Panamá. Centros, 3(2): 121-142.

Santos, M. A. Yostin, A. R. 2016. Contribución al conocimiento de la diversidad de abejas de las orquídeas (apidae: euglossini) de la península de Azuero, Panamá, Tecnociencia, 18(2): 45-58.

Santos, M. A., Oscar, G. L., y Ronald, S. A. 2011. Estratificación vertical de las abejas de las orquídeas (Euglossinae) en un bosque tropical húmedo, monte fresco, Cerro Azul, Panamá. Tecnociencia, 13(2): 99-108.

Santos, M. A., Muñoz, M. R., Medianero, E., Osorio, M., y Carranza, R. 2012. Abejas de las orquídeas (Hymenoptera: Apidae: Euglossini) en la comarca Guna de Madugandí provincia de Panamá, Panamá. Scientia, 22(2): 77-91.

Santos Murgas A., Abrego L. J., Añino R. Y., López Ch. O. G. 2016. Notas sobre depredación de Apiomerus hirtipes (Hemiptera: Reduviidae) sobre abejas de la orquídea (Apidae: Euglossinae). Revista Científica Centros (Panamá) vol. 5(2): 46-52.

Santos Murgas, A., Ábrego L. J., López Ch. O. G., Monteza M. C. M., Osorio A. M. A., Guardia Q. R., Álvarez H. E. A., Quiroz K., Añino R. 
Y. J., Carranza B. R. E. \& Villarreal C. 2018. Abejas de las orquídeas (Hymenoptera: Apidae) del Parque Nacional Darién, Panamá. Tecnociencia vol. 20 (2): 59-69.

Silva, F. S., y Rebêlo, J. M. 2002. Population dynamics of Euglossinae bees (hymenoptera, Apidae) in an early second-growth forest of cajual island, in the state of Maranhão, Brazil. São Luís, Brazil. Braz. J. Biol, $62(1): 15-23$.

Tonhasca, J. R., Blackmer J. L., y Albuquerque G. S. 2000. Euglossine bees as indicators of the conservation status of Atlantic forest fragments in Rio de Janeiro State, Brazil. Brazil. Coservation ecology comment.

Veiga, S. N., y Barbosa, G. R. 2015. Is the capture success of orchid bees (Hymenoptera, Apoidea) influenced by different baited trap designs? A case study from southern Brazil. Revista Brasileira de Entomologia. 59: 32-36.

Walker, W. 2005. The strengths and weaknesses of research design involving quantitative measures. Res Nurs 10(5): 571-81.

Recibido 3 de marzo de 2020, aceptado 28 de abril de 2020. 


\section{Anexos}

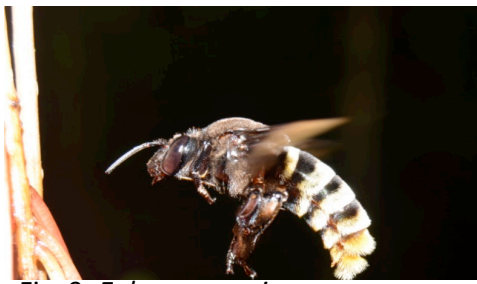

Fig. 9. Eulaema meriana

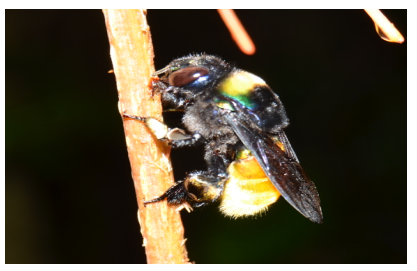

Fig. 11. Eufriesea

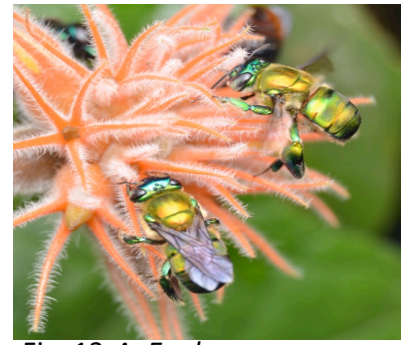

Fig. 13-A. Euglossa sp

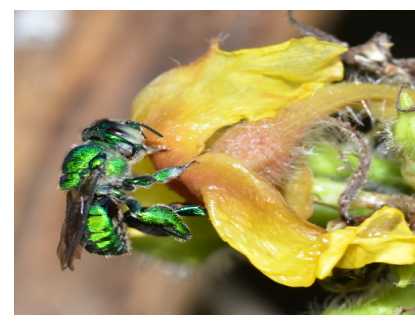

Fig. 13-C. Euglossa sp

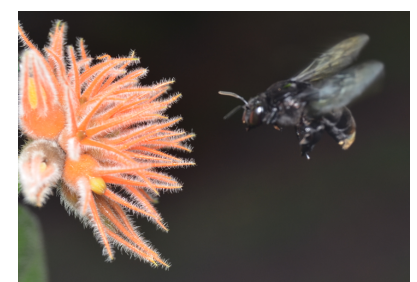

Fig. 10. Eulaema nigrita

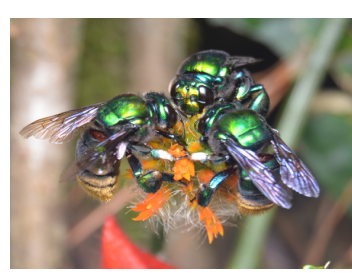

Fig. 12. Eufriesea anisochlora C

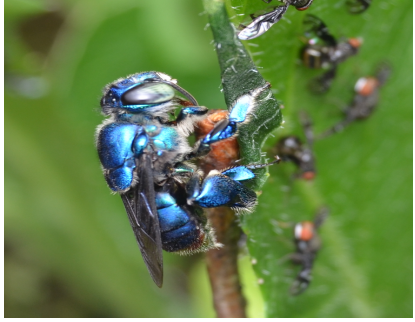

Fig. 13-B. Euglossa sp

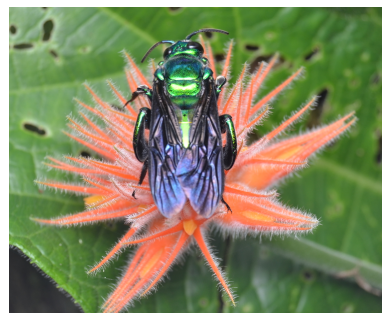

Fig. 14. Exaerete frontalis

Tecnociencia, Vol. 22, $N^{\circ} 2$ 